\title{
Kernos
}

Revue internationale et pluridisciplinaire de religion grecque antique

12| 1999

Varia

\section{La mémoire et la mort dans l'épopée homérique}

\section{David Bouvier}

URL : http://journals.openedition.org/kernos/708

DOI : $10.4000 /$ kernos.708

ISSN : 2034-7871

\section{Éditeur}

Centre international d'étude de la religion grecque antique

Édition imprimée

Date de publication : 1 janvier 1999

Pagination : 57-71

ISSN : 0776-3824

Référence électronique

David Bouvier, "La mémoire et la mort dans l'épopée homérique », Kernos [En ligne], 12 | 1999, mis en ligne le 13 avril 2011, consulté le 30 avril 2019. URL : http://journals.openedition.org/kernos/708 ; DOI : 10.4000/kernos.708 
Kernos, 12 (1999), p. 57-71.

\section{La mémoire et la mort dans l'épopée homérique}

\section{L'île d'oubli}

Que peut-il en être de la double fonction de la mémoire et de l'oubli dans la vie d'une société ? La formation de l'identité collective implique-t-elle des procédures particulières de commémoration ou des stratégies concertées d'oubli ? En quoi la mémoire et l'oubli peuvent-ils contribuer à souder une collectivité ${ }^{1}$ ?

S'agissant de la Grèce archaïque, faute de pouvoir travailler directement sur le terrain, comme le ferait l'ethnologue ou le sociologue contemporain, je me contenterai des textes. La réalité de la société grecque archaïque n'est jamais directement accessible, elle ne peut être que reconstruite d'après une série de conjectures inspirées par des témoignages de natures diverses. Pour la présente enquête, je m'en tiendrai aux poèmes homériques et j'irai chercher mon premier témoignage dans ce monde à part, que les Anciens euxmêmes définissaient comme un monde fictif et qui est décrit dans ces récits

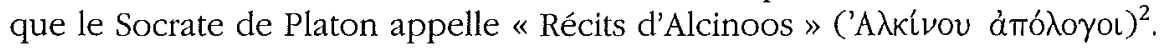

Dans l'Odyssée, après dix ans d'errance, Ulysse échoue enfin sur le rivage de Phéacie où il est accueilli à la cour du roi Alcinoos. Là, le deuxième soir, il est invité par ses hôtes à révéler son identité et à raconter les péripéties de

1 Sur la dimension politique de l'oubli, $c f$. N. Loraux, L'oubli dans la cité, in TR, 1 (1980), p. 213-242; De l'amnistie et de son contraire, in Y.H. Yerushalmi \& al. (éds), Usages de l'oubli, Paris, 1988, p. 23-47; Le 2 Boèdromion. Á propos d'un jour interdit de calendrier à Atbènes, in P. Gignoux (éd.), La commémoration, Paris - Louvain, 1988, p. 57-72; ces deux études sont reprises dans N. Loraux, La cité divisée, Paris, Payot, 1997, p. 11-40 et p. 146-194. Voir aussi maintenant M. Sordi (éd.), Amnistia, perdono e vendetta nel mondo antico, Milano, Vita e Pensiero, 1997 (Pubblicazioni dell'Universitá Cattolica del Sacro Cuore), ainsi que mes remarques dans Mémoire poétique et politiques d'oubli en Grèce antique, in Traverse. Revue d'Histoire (1991/1) [= Non-lieux de mémoire], p. 49-58.

2 Platon, Rép., 614b 1; Arist't., Poét., 1455a 2 et Rbét., 1417a 13. Sur la conception de la mémoire et de l'oubli dans la littérature grecque, $c f$. surtout J.-P. VERnANT, L'individu, la mort, l'amour. Soi-même et l'autre dans la Grèce ancienne, Paris, 1989, p. 41-115, à qui cette étude doit beaucoup, et M. Simondon, La mémoire et l'oubli dans la pensée grecque jusqu'à la fin du ve siècle av.J.-C., Paris, 1982. Sur le processus de laîcisation de la mémoire en Grèce ancienne, $c f$. D. BOuviER, "Mneme ». Le peripezie della memoria greca, in S. Settis (éd.), I Greci. 2 II: Una storia greca: Definizione, Torino, Einaudi, 1997, p. 11311146. 
son voyage. Ulysse se nomme et commence à raconter son périple : le départ de Troie, l'étape au pays des Cicones et la tempête au large du cap Malée qui entraîna son vaisseau dans un monde de nulle part, fondamentalement différent du monde humain. Dans cet univers de l'ailleurs, Ulysse et ses compagnons font une première escale, après neuf jours de tempête, au pays des Lotophages, véritable île d'oubli. C'est la première étape d'Ulysse dans le monde de l'inconnu. Le héros raconte comment il envoya alors, prudemment, trois de ses hommes explorer l'île.

Aussitôt partis, les voilà qui vont se mêler aux Lotophages. Ceux-ci ne trament rien de mal contre nos compagnons mais leur offrent à goûter du lôtos. Et dès que l'un d'eux goûte à ce fruit du lôtos aux saveurs de miel, il ne

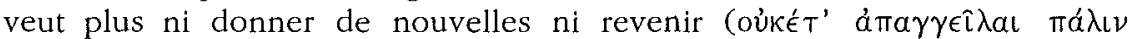

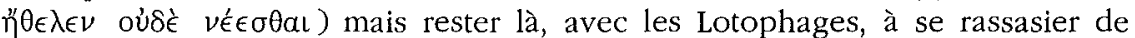
lôtos en oubliant le retour (vóotov $\tau \epsilon \lambda \alpha \theta \dot{\epsilon} \sigma \theta a \mathrm{~L}$ ). Je dus les ramener de force à nos vaisseaux bien taillés, tandis qu'ils pleuraient, et, en les tirant, les attacher sous les bancs. J'ordonnai au reste de mon fidèle équipage d'embarquer avec hâte sur les nefs rapides de peur qu'un autre, ne goûtant au lôtos n'oubliât à son tour l'heure du retour $\left(O d\right.$., IX, 91-102) ${ }^{3}$.

Curieuse société que celle des Lotophages, un peuple dont on ne saura rien d'autre que son penchant pour le lôtos, ce fruit d'oubli, qui constitue l'essentiel de sa nourriture ${ }^{4}$. Sur le régime politique des Lotophages, sur leurs croyances religieuses, leurs institutions, leurs arts et métiers, Ulysse ne dit rien, comme si les Lotophages n'avaient d'autre occupation que de consommer du lôtos. De fait, lorsqu'ils rencontrent les compagnons d'Ulysse, les Lotophages n'ont d'autre réaction que de leur offrir, en guise d'accueil, un déjeuner de lôtos. C'est une première caractéristique des Lotophages, ils sont accueillants; ils respectent l'usage qui veut qu'on offre aux hôtes et aux visiteurs un repas d'accueil ${ }^{5}$. Mais ce repas est tel qu'il transforme son consommateur en Lotophage. Manger du lôtos, c'est ne plus vouloir se rappeler aux siens, ne plus vouloir donner aux autres la moindre nouvelle de soít; c'est oublier sa propre origine et sa propre identité pour devenir Lotophage et vivre d'oubli.

Si brièvement racontée qu'elle soit, l'étape chez les Lotophages n'en met pas moins en évidence la force de l'oubli comme facteur de cohésion sociale.

3 Pour cette citation comme pour les suivantes, je donne mes propres traductions.

4 A. Heubeck (Commentary on Homer's Odyssey: II: Books 9-12, Oxford, 1989, p. 18, ad loc.) tend à penser que les Lotophages mangeaient exclusivement du lôtos. Sur cet épisode, $c f$. aussi M. Rosseaux, Ulysse et les mangeurs de coquelicots, in BAGB, 71 (1971), p. 333-351.

5 Comme l'a relevé G. Germain, Genèse de l'Odyssée. Le fantastique et le sacré, Paris, 1954, p. 225.

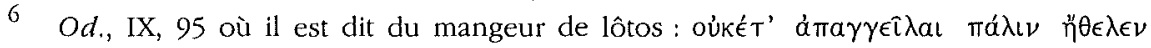

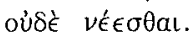


Les Lotophages sont une société sans violence, sans classe sociale, sans distinction culturelle, sans héros non plus : une société où personne ne se soucie de se faire entendre et reconnaître dans sa différence. Ici, chacun accepte d'être comme l'autre : un mangeur de lôtos; le banquet d'oubli unifie le groupe des convives autant qu'il le rassemble. Dans cette société où l'autre devient même, aucun échange culturel n'est possible; l'étranger est accepté sans la moindre réticence, il est de toute manière condamné à devenir, comme les autres, un Lotophage. Défait de sa mémoire individuelle, oublieux de sa propre patrie, le visiteur étranger ne peut, à la table des mangeurs de lôtos, que s'assimiler aux autres. Partageant une même nourriture d'oubli, la société des sans mémoire est une société sans différence ni différent possibles. Pas de lutte contre l'étranger, pas d'affrontement entre les classes, pas de guerre interne; l'oubli efface les différences d'origine et de culture, il dissipe revendications et plaintes, et abolit ambitions et désirs de conquête.

À plus d'un égard, la communauté des Lotophages apparaît comme une société idéale, parfaitement soudée, sans risque aucun de division. Plus que la mémoire, l'oubli semble pouvoir contribuer au bonheur de la société. Les compagnons d'Ulysse pleurent, en effet, lorsque leur chef les arrache à leur nouvelle vie pour les ramener de force à leur nef ${ }^{7}$. Faut-il alors penser que l'oubli est le secret d'une société idéale tandis qu'une mémoire absolue serait un facteur de division contribuant à faire éclater la société ? L'exemple des Lotophages permet, au moins, de poser la question ${ }^{8}$. Mais l'essentiel est qu'Ulysse n'envisage pas un instant de s'attarder dans cette île d'oubli. Si la société des sans mémoire existe, elle est sur le chemin du héros grec une étape interdite. Le bien-être du peuple oublieux est aussi une négation des valeurs héroïques.

\section{L'île de la toute mémoire}

Après l'épisode des Lotophages, Ulysse continue le récit de ses aventures. Parmi les derniers dangers que le héros doit affronter, il y a le chant des Sirènes, ce chant qui est riche d'une mémoire absolue. C'est du moins ce que prétendent les Sirènes, quand elles voient passer au large la nef d'Ulysse :

Viens ici, Ulysse aux mille louanges, gloire éternelle des Achéens, arrête ta nef et écoute le concert de nos deux voix. Jamais personne n'a laissé son noir navire dépasser notre cap sans écouter couler de nos bouches la mélodie de nos voix; mais après avoir subi notre charme, il s'en est retourné plus riche en savoir. Car nous savons tout ce que les Troyens et les Achéens ont souffert,

7 Od., LX, 98-99.

8 Une enquête plus poussée gagnerait à réfléchir aux pouvoirs respectifs du lôtos et de la drogue de bien-être employée par Hélène en $O d$., IV, $220 s q$. Voir aussi cet oubli que Zeus impose aux gens d'Ithaque en Od., XXIV, 484-485; à ce propos, $c f$. Loraux, $o p$. cit. (n. 1), p. 157-160. 
de par la volonté des dieux, dans la vaste Troie; nous savons tout ce qui arrive sur la terre riche en nourritures (Od., XII, 184-191).

Une promesse de savoir absolu. Mais Circé avait averti Ulysse sur l'envers de l'offre:

Tu arriveras d'abord chez les Sirènes qui charment tous les hommes, quels qu'ils soient à les approcher. Celui qui s'arrête, insouciant, pour écouter leur voix, celui-là ne voit plus sa femme et ses jeunes enfants l'accueillir et fêter son retour. Car les Sirènes le tiennent sous le charme de leur chant clair, assises dans une prairie, tandis qu'autour d'elles, le rivage est jonché d'os, de corps qui pourrissent et de chairs putréfiées. Passe ta route sans halte et bouche les oreilles de tes compagnons avec de la cire de miel pétrie, afin qu'aucun d'eux ne puisse entendre. Toi-même, si tu le veux, écoute mais, dans ta nef rapide, debout, fais-toi attacher pieds et mains au mât; là, reste attaché aussi longtemps que tu entendras l'envoûtante voix des deux Sirènes. Et si tu supplies et exhortes tes compagnons de te délier, qu'ils t'attachent avec plus de nœuds encore (Od., XII, 39-54).

Pour le héros de l'Odyssée, l'étape chez les Sirènes est aussi déconseillée que celle chez les Lotophages. Cette fois, ce n'est plus à Ulysse d'attacher ses compagnons aux bancs de la nef mais à eux de le lier, bien solidement, au mât. Ici, comme chez les Lotophages, le voyageur qui voudrait s'attarder s'arrêterait pour toujours. Les Sirènes, à la voix parfaite, connaissent un chant qui sait tout; elles savent tout ce qui est advenu et tout ce qui advient dans le monde des hommes ${ }^{9}$. Un savoir absolu qui implique une mémoire absolue. Mais ce savoir complet est dangereux pour l'homme; plus qu'il n'enseigne, il enchante, pareil finalement - c'est là le paradoxe - à une drogue d'oubli. Celui qui s'arrête pour écouter les Sirènes ne repart plus mais veut rester là à écouter jusqu'au bout le chant qui sait tout, inconscient du danger qu'il y a, pour un mortel, à vouloir s'attarder trop longtemps chez des immortelles. Le décor décrit par Circé laisse deviner une forte contradiction entre la condition humaine et le savoir total. Pour tout entendre du chant qui sait tout, il faudrait plus qu'une vie humaine. Des derniers auditeurs jadis charmés par les Sirènes, il ne reste maintenant plus que des carcasses d'os et de chairs putréfiées $^{10}$. Dans la société des mortels, la mémoire est un moyen de voyager dans le temps, de faire reviure les ancêtres et d'établir ainsi un lien entre les gériérations qui se succèdent. Dans le cas des Sirènes, les choses sont très différentes; englobant tout, leur savoir suppose une forme d'éternité. Alors que la mémoire des hommes se déplace dans le temps, celle des Sirènes le contient tout entier. Pour tout entendre de leur chant, il faudrait l'entendre

9 Sur le chant des Sirènes, cf. surtout P. Pucci, The Song of the Sirens, in Aretbusa, 12 (1979), p. 121-132 et Ulysse Polutropos, Lectures intertextuelles de l'Iliade et de l'Odyssée, Lille, 1995 [or, angl. 1987], p. 288-293.

10 Sur le sens de ce charnier, cf. J.-P. VernanT, Le refus d'Ulysse, in TR, 3 (1982), p. 15, et op. cit. (n. 2), p. 115. 
éternellement. Le savoir des Sirènes exige la totalité du temps : il est, à ce titre, un tout indivisible, inaccessible à l'homme.

En allant de l'île des Lotophages à celle des Sirènes, Ulysse est passé de la séduction d'oubli extrême à la séduction de mémoire absolue. On pourrait s'étonner de ce héros qui renonce aussi bien à l'innocence heureuse des mangeurs de lôtos qu'au charme du savoir total. Chez les Sirènes aussi bien que chez les Lotophages, quelque chose est déréglé qui déshumanise ces lieux et interdit au héros de s'y attarder ${ }^{11}$. Pour comprendre ce qui est ici en cause, il importe d'examiner plus directement comment l'Iliade et l'Odyssée définissent le rôle réciproque de la mémoire et de l'oubli dans la société des héros.

\section{Les fantômes sans mémoire de l'Hadès}

Entre son escale chez les Lotophages et sa rencontre avec les Sirènes, Ulysse a dû se rendre au seuil de l'Hadès pour y consulter l'âme de Tirésias qui, seule, peut lui révéler l'itinéraire de son retour à Ithaque. Dans le monde de nulle part, cette étape a une valeur particulière; elle permet au héros de découvrir, non plus un monde qui s'oppose à celui des hommes, mais une réalité qui est la nécessaire contrepartie de la vie humaine et des valeurs qui lui sont attachées.

Enveloppé de brumes que les rayons du soleil ne transpercent jamais ${ }^{12}$, le

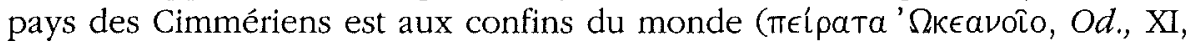
13), " là où l'Achéron reçoit les eaux du Pyriphlégéthon et du Cocyte qui coule du Styx » $(\mathrm{X}, 513-4)$. Cette ultime frontière de la terre est aussi le lieu privilégié d'une rencontre impossible : en ce bout du monde, les vivants peuvent invoquer et rencontrer les morts. Précisons bien : Ulysse ne se rend pas au pays des morts mais en un point frontière où vivants et morts peuvent se retrouver. Arrivé au pays des Cimmériens, le héros accomplit le rituel d'invocation que Circé lui a prescrit; il sacrifie un agneau et une brebis noire cependant qu'autour de lui se rassemble le peuple des morts. Si Ulysse peut alors reconnaître et identifier l'âme de chacun des défunts, les âmes sont, elles, incapables de le reconnaître et de s'adresser à lui aussi longtemps qu'elles n'ont pas bu un peu du sang des victimes sacrifiées ${ }^{13}$. Dans l'Hadès, les morts existent sans exister : ils ne sont que des «fantômes » $\left(\epsilon^{\prime \prime} \delta \omega \lambda \alpha\right)$, des « têtes

11 Sur la menace, parallèle à celle des Sirènes, qu'incarnent dans l'Odyssée Calypso et Circé, $c f$. D. Bouvier, L'aède et l'aventure de mémoire, in Ph. Borgenud (éd.), La Mémoire des Religions, Genève, 1988, p. 73-76.

12 Od., XI, 15-16.

13 Ainsi, l'âme d'Anticléia ne reconnaît son fils que lorsqu'elle a goûté au sang (od, XI, $140 s q$.). Seul Tirésias conserve sa conscience et sa mémoire parmi les morts. Sans expliquer le pourquoi de cette exception, on peut noter que ses facultés de devin et sa condition d'aveugle faisaient de lui, avant la mort déjà, un vivant différent des autres, lié, de son vivant même, à l'univers de l'invisible qui inclut l'Hadès. 


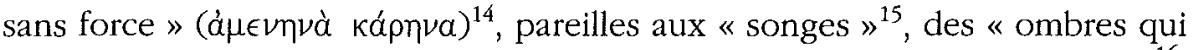

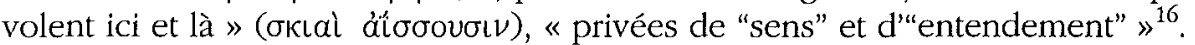
La condition des morts est si peu de chose que jamais on ne voit les héros s'inquiéter véritablement de ce qui les attend dans l'au-delà. De leur condition de morts, réduits à ne plus être que des fantômes sans mémoire ni sensation, les héros n'espèrent ni ne redoutent rien; jamais, on ne les voit chercher dans l'après-vie l'explication de leurs souffrances terrestres ${ }^{17}$. Il n'y a rien dans l'Hadès qui puisse apporter un sens ou une justification aux peines et aux souffrances de la vie terrestre. Pas d'enfer pour punir, ni de paradis pour récompenser mais seulement un lieu obscur et invisible où flottent des images sans consistance.

Entre la société des morts et celle des Lotophages, il existe un point commun : dans les deux cas, on a affaire à une communauté sans mémoire. Mais ce point commun ne fait qu'accentuer le statut paradoxal des Lotophages qui, vivants, sont alors comme des morts. Que peut-il en être d'une opposition de la vie et de la mort dans un tel cas? En quoi les valeurs de la vie pourront-elles se démarquer de celles de la mort? Dans le monde hérö̈que, l'oubli absolu est une caractéristique de la mort; en tant que tel, il revêt un sens bien précis qu'il ne saurait avoir chez les Lotophages.

\section{La mémoire comme réponse à la mort dans la poésie ho- mérique}

Examinons un point particulier. Au pays des Cimmériens, quand Ulysse accomplit son sacrifice aux morts, la première ombre qui se présente à lui est celle de son compagnon Elpénor, qui aurait dû l'accompagner dans ce voyage s'il n'avait fait, juste avant l'embarquement, une chute mortelle. $\grave{A}$ Ulysse, l'âme d'Elpénor adresse une prière bien précise; dans l'urgence du départ, personne ne s'est avisé de sa mort et son corps est resté dans l'île de Circé, privé de funérailles et de sépulture :

Maintenant, je t'en supplie, au nom de ceux que tu as laissés derrière toi et qui ne sont pas ici, au nom de ton épouse et de ton père qui te nourrit quand tu étais enfant, au nom de Télémaque, ce fils unique que tu as laissé dans ton manoir! Je sais, en effet, que, lorsque t'en allant d'ici tu quitteras la demeure d'Hadès, tu conduiras ton vaisseau bien construit à l'île d'Aiaié [l'île

14 Od., X, 521 et XI, 29.

15 Od., XI, 207.

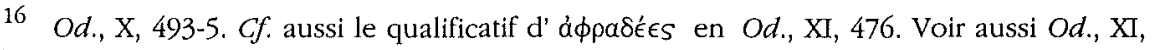
216-223; 488-91 et $I l$. XXIII, 104. La discussion entre les âmes des défunts au début du chant XXIV de l'Odyssée («Deuteronekuia ») fait ici problème; on rappellera toutefois que ce passage, hautement problématique, était déjà considéré par les Anciens comme une absurde interpolation.

17 Sur l'opposition du héros homérique et du héros chrétien, $c f$. E.R. Dodds, Les Grecs et l'irrationnel, Paris, 1977 [or. angl. 1951], p. 28. 
de Circé où se trouve le corps d'Elpénor]. Quand tu seras de nouveau là-bas,

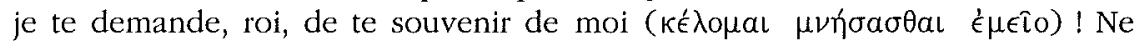
repars pas en me laissant sans pleurs, sans funérailles de crainte que je n'attire sur toi la colère des dieux $(\theta \in \hat{\omega} \nu \mu \eta \dot{v} \nu \mu a)$. Mais brûle-moi avec toutes mes armes et dresse, sur le rivage de la mer argentée, la tombe d'un homme mal-

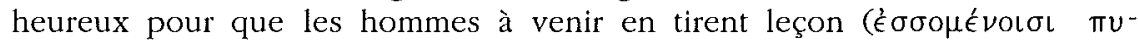
$\theta \dot{\epsilon} \sigma \theta a \mathrm{l}$ ). Cela, fais-le pour moi et sur ma tombe ( $\tau u ́ \mu \beta \omega)$, fixe la rame que je maniais, quand, vivant, j'étais avec mes compagnons ( $O d$., XI, 66-78).

Ce passage peut être rapproché d'un épisode de l'Iliade où l'on voit l'âme de Patrocle s'adresser à Achille. La scène se passe au chant XXIII. Dans son obstination à honorer Patrocle, Achille ne fait que prolonger exagérément les funérailles de son ami. Il a d'abord capturé douze jeunes Troyens dans l'intention de les égorger sur le bûcher funéraire; il a ensuite tué Hector pour réserver à son cadavre les pires outrages. Épuisé par cette cuvre de vengeance, Achille se laisse bientôt vaincre par le sommeil. Pendant qu'il dort, voilà que se présente à lui l'âme de Patrocle, toute pareille à ce qu'était le héros de son vivant; se dressant au-dessus de sa tête; elle lui parle:

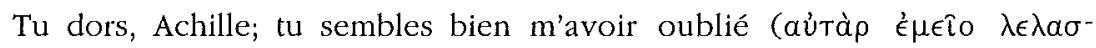
$\mu \epsilon^{\prime}{ }^{\prime}{ }^{\prime \prime} \pi \lambda \epsilon U$ ); tu prenais soin du vivant mais non du mort. Ensevelis-moi le plus vite possible; je veux passer les portes de l'Hadès ! Les âmes, fantômes des défunts, me repoussent loin à l'écart, sans me laisser franchir le fleuve pour les rejoindre. J'erre ainsi en longeant la demeure d'Hadès aux larges portes. Donne-moi la main, je te le demande en pleurant; car je ne reviendrai plus de l'Hadès quand vous m'aurez donné ma part de feu. [...] Mais, si tu m'écoutes, je veux te dire et te recommander encore autre chose : Achille, ne dépose pas mes cendres loin des tiennes, mais place-les ensemble; nous avons grandi ensemble dans ta maison [...] Ainsi donc qu'une même urne recèle nos cendres à tous deux, ce vase d'or que t'offrit ta vénérable mère ( $I l$., XXIII, 69-92).

Patrocle et Elpénor ont ici en commun de n'avoir pas encore reçu, au moment où ils parlent, la tombe qui leur revient. Contrairement aux âmes des autres défunts, ils ont aussi en commun d'être encore en pleine possession de leur mémoire et de leur conscience. Ils n'ont nul besoin de se rassasier de sang pour s'entretenir avec les vivants. Morts, mais détenant encore leurs facultés intellectuelles de vivants, ils se trouvent dans cette situation paradoxale qui leur interdit de rejoindre les autres défunts alors même qu'ils ont cessé de vivre. Privés de tombe, repoussés par la foule des âmes sans mémoire, « oubliés » ${ }^{18}$ par des vivants qui tardent à leur accorder leur tombe, ils errent dans une zone intermédiaire. Tous deux revendiquent, en fait, leur droit à un $\sigma \hat{\eta} \mu a$, cette tombe que les Grecs appelleront, après Homère, un 
$\mu \nu \eta \hat{\mu} \alpha$, un « mémorial ${ }^{19}$. Patrocle le dit très nettement : pour passer les portes de l'Hadès, il doit d'abord avoir été enseveli par les vivants, avoir reçu d'eux la tombe qui fera de lui une figure digne d'être commémorée. Elpénor dit la même chose mais d'une autre façon: si Ulysse ne lui donne pas la sépulture qu'il mérite, il risque de devenir pour lui « la cause d'une colère divine ». C'est une faute religieuse pour un vivant que de ne pas accorder au mort la tombe qu'il mérite ${ }^{20}$. Patrocle et Elpénor expriment, tous deux, leur désir de passer dans l'Hadès où ils seront déchargés de leur mémoire et de leur conscience. Comme si le souvenir de la vie devenait, dans la mort, gênant et trop lourd à porter. Pour le mort, la mémoire est un défaut, elle le rattache paradoxalement à un monde qu'il a irrémédiablement quitté. Toutefois, si Patrocle et Elpénor ont hâte de passer les portes de l'Hadès, ce n'est pas pour devenir des âmes sans conscience ni mémoire mais parce que cette absence de conscience est une condition sine qua non pour accéder à une autre forme de survie : celle qui fera du mort une figure exemplaire, digne de survivre dans la mémoire des vivants.

Si le héros homérique ne se soucie pas de ce qu'il deviendra dans l'Hadès, il s'inquiète, en revanche, grandement de la survie de son nom et de sa renommée dans le monde des vivants. C'est dans la mémoire des hommes à venir et nulle part ailleurs que le héros veut survivre et être immortalisé. Il est prêt pour cela à payer de sa vie l'exploit qui le rendra éternellement mémorable. Dans le monde homérique, la mort ne peut être véritablement transcendée et dépassée que sur le mode de la mémoire. La poésie des aèdes qui célèbre l'histoire des ancêtres, la tombe qui rappelle l'existence du mort, les noms donnés aux descendants mais qui renvoient à l'identité des pères, toutes ces pratiques apparaissent comme autant de procédures de mémoire destinées à perpétuer le souvenir du défunt pour qu'il continue ainsi d'exister dans le monde des vivants, où sa présence physique est devenue impossible $^{21}$. On peut citer ici ce moment essentiel de l'Iliade, au chant XXII, lorsque a lieu le duel d'Achille et d'Hector. Devant un ennemi plus fort que lui, certain de devoir mourir, Hector n'en accepte pas moins le combat sans nullement chercher à fuir. Plus que sa vie, il entend sauver son honneur :

Elle est maintenant toute proche l'affreuse mort qui m'attend, là tout à côté, sans moyen d'y échapper. [...] Le destin m'a ainsi rattrapé. Mais, je ne

19 Simondon, op. cit. (n. 2), p. 85 sq. On notera qu'en allemand, le terme Denkmal associe aussi les idées de tombe et de mémorial,

20 Sans être une figure de premier plan et malgré sa mort si peu hérö̈que, Elpénor n'en reste pas moins un héros. On notera toutefois la différence d'importance entre la figure d'Hector et celle d'Elpénor. L'Odyssée se plaît ici, une fois encore, à remettre en cause l'idéologie de l'Iliade. Dans la présente étude, pour des raisons de clarté, je n'ouvre pas le dossier d'une Odyssée critiquant l'Iliade. Voir toutefois Pucci, op. cit. (n. 9), p. 36.

21 Sur ces différentes procédures, H. MüHLEstein, Homerische Namenstudien, Frankfurt am Main, 1987, p. 28 sq. et J. Svenbro, Phrasikleia. Anthropologie de la lecture en Grèce ancienne, Paris, 1988, p. 90. 
veux pas mourir sans me battre et sans gloire; je veux accomplir un geste de

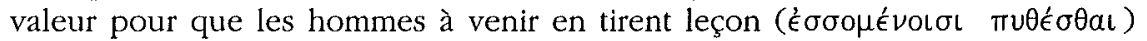
(Il., XXII, 299-305).

Les mots d'Hector sont ici les mêmes que ceux d'Elpénor : ne pas mourir sans laisser une marque de soi pour que les autres puissent se souvenir, pour que puisse être établi, entre le mort et les hommes de demain, le lien de mémoire $^{22}$. Le héros ne peut survivre à sa condition de mortel qu'en tant que sujet de mémoire.

\section{Un pays où l'on oublierait les morts}

Dans la poésie archaïque, mais cela est vrai de manière plus générale, la mort pose le problème de la solidarité et de la cohésion sociales. Comment une société peut-elle rester elle-même si chacun des membres qui la compose est condamné, un jour ou l'autre, à mourir et à disparaître? Il y a ici un paradoxe qui renvoie à la contradiction même de la vie et de la mort. Comment accepter que ce qui était là puisse soudain ne plus être là ${ }^{23}$ ? Prenons l'exemple du cadavre. Encore présent au milieu des vivants, encore exposé à une lumière qui fut pour lui source de vie ${ }^{24}$, le corps du mort devient l'objet d'une inquiétante transformation: encore visible, il est condamné à se

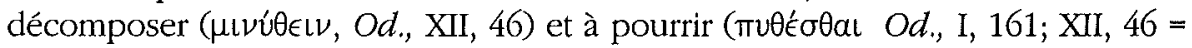
Il., XI, 395); la lumière du soleil qui faisait vivre le vivant dessèche le mort, le vide de sa fraîcheur et précipite sa putréfaction et son passage dans l'invisible. Comment accepter que celui qui était là et qui appartenait pleinement au groupe soit soudain condamné à se décomposer pour disparaître bientôt complètement? Face au spectacle bien réel de cette disparition, la société doit inventer une stratégie de récupération qui permette de réintégrer le mort "symboliquement». Les funérailles et le tombeau trouvent ici tout leur sens. Les funérailles sont d'abord l'occasion pour le groupe des vivants de se réunir et de se retrouver autour du défunt. En lui accordant un nouveau statut social, en reconnaissant en lui une figure digne d'être commémorée, les vivants choisissent de faire vivre le mort en eux. Le mort n'est plus ce cadavre qui va disparaître dans le vide mais une figure qui s'inscrit dans la mémoire de chacun et dont chacun cherchera à partager les qualités exemplaires. Autour de la tombe offerte au mort, le groupe réaffirme son unité au nom d'un système de valeurs partagées par tous et symbolisées par le mort. C'est face au mort et autour de lui que le groupe des vivants peut finalement - para-

22 Cf. ici les remarques importantes de J.M. REDFIELD, Nature and Culture in the Iliad, The Tragedy of Hector, Durham - London, $1994^{2}$ [1975], p. 34 ainsi que VERnant, op. cit. (n. 2), p. 70 .

23 Sur ce problème, $c f$. aussi Vernant, op. cit. (n. 2), p. 105.

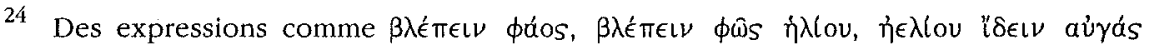
prennent le sens de « vivre », cf. Esch., Perses, 261; Eur., Hél., 60; Il., XVI, 188, Od., XI, 498; 619 , etc. 
doxalement - réaffirmer le mieux la cohésion de son unité. La tombe est aussi symbole de solidarité sociale. Le rivage de l'île des Sirènes jonché d'ossements et de chairs putréfiées est au contraire, dans la poésie homérique, une image effrayante, parfaite négation de tout ce qui constitue une société civilisée : un lieu on l'on ne sait pas encore se souvenir des morts.

Pour expliciter ce point, on peut se plonger plus avant dans l'un des multiples effets de miroir de l'Odyssée. Au début du premier chant, Télémaque confie à son hôte, Mentès, toute l'inquiétude que lui cause l'absence d'un père qu'il attend depuis trop longtemps; il craint qu'il ne reste plus de ce père jamais vu que des « os blancs pourrissant à la pluie, échoués sur quelque rivage, ou emportés au large par le ressac des vagues» (I 161-162). Sans le savoir, Télémaque redoute l'éventualité d'un Ulysse qui se serait trop longtemps attardé dans l'île des Sirènes ${ }^{25}$. Aux cadavres privés de sépulture, il oppose très nettement la tombe que les Achéens auraient donnée à Ulysse s'il était mort dans la plaine de Troie, ou celle qu'il aurait reçue de ses proches s'il était mort près d'eux à Ithaque. Au mort mis en terre et qui lègue aux siens un prestigieux renom, $\kappa \lambda \hat{\text { ós}}$, Télémaque confronte le sort d'un être disparu sans laisser aux siens aucune gloire ni nouvelle ${ }^{26}$; d'un côté la réalité d'une tombe visible, de l'autre le vide laissé par un homme qui aurait pu, tout aussi bien, n’avoir jamais existé.

Désormais les dieux, aux vilains desseins, en ont voulu autrement; ils ont fait de lui le plus invisible (ăiotov) des hommes; je ne souffrirais pas autant s'il était mort, tombé au milieu de ses compagnons au pays des Troyens, ou dans les bras des siens, une fois la guerre finie. Les Panachéens lui auraient fait une tombe, il aurait gagné, pour me la transmettre, une grande gloire

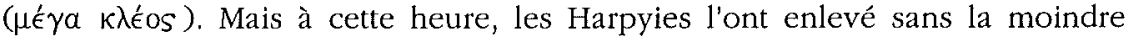
gloire $(\alpha \dot{\alpha} \lambda \epsilon \in \omega \hat{s})$; il est parti invisible, sans qu'on puisse rien savoir de lui

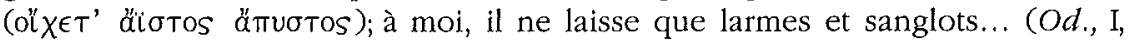
234-243).

Les propos de Télémaque sont ici fondamentaux, ils révèlent tout le sens des funérailles et du tombeau dans le monde des héros. La mort devient acceptable dès lors que les vivants peuvent accorder au défunt la tombe qui lui revient et qui signifie son nouveau statut social. Mais pour que cela soit, il faut que le héros soit mort parmi ses compagnons d'armes ou parmi ses proches; il faut que l'on sache comment il est mort. C'est là un point essentiel : dans le monde archaïque, l'individu se révèle avant tout dans sa façon

25 Comparer Od., I, 161-162 et XII, 45-46. Sur le statut particulier de la figure de Télémaque dans l'Odyssée, cf. R.P. MARTIN, Telemacbus and the Last Hero Song, in Colby Quarterly, 29 (1993), p. 222-240.

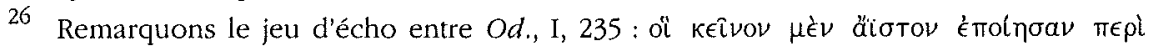

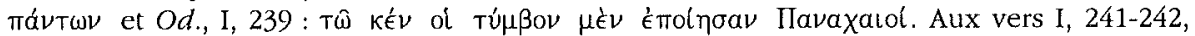
Télémaque revient sur ce thème d'un Ulysse devenu ăioros et ämuotos, enlevé sans

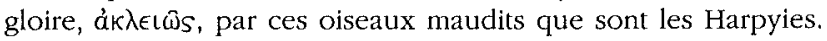


d'affronter la mort. Or Télémaque - et c'est là ce qui le chagrine le plus - ne sait rien de l'éventuelle mort de son père. Phémios, l'aède d'Ithaque, qui chante les retours des héros, est, depuis longtemps, contraint par les prétendants de chanter ce qu'ils veulent entendre: un chant tout à la honte d'Ulysse. Télémaque craint non pas qu'on oublie son père mais bien qu'on le calomnie. Tant qu'il ne saura pas la manière dont son père a affronté la mort, tant qu'il ne saura pas ce que ce père est devenu, il ne saura pas le crédit qu'il faut accorder aux insinuations infamantes des prétendants. Rien ne garantit à Télémaque qu'Ulysse est encore - à l'heure où il pleure - un héros irréprochable, rien n'exclut que ce père, auquel il ne cesse de penser, ait pu commettre quelque acte indigne de lui et souiller, ainsi, la réputation du clan familial ( $\gamma$ évos) tout entier.

Cette absence d'informations sur Ulysse constitue pour Télémaque un véritable problème. Dans la société homérique, un fils doit être à l'image de son père, pareil à lui. Au sein du yévos, l'identité est héréditaire : le fils reçoit « de qui tenir » et doit prouver, par son comportement, qu'il est bien le descendant de ses ancêtres, qu'il mérite d'appartenir au $\gamma$ '́vos qui est le sien. Ignorant du sort d'Ulysse, privé de toute nouvelle à son sujet, Télémaque voit sa propre identité remise en question ${ }^{27}$. Aussi longtemps qu'il ne saura rien de son père, il restera incertain de sa propre identité. Le pire serait pour lui qu'Ulysse ait, effectivement, définitivement disparu.

Pour les vivants, il est fondamental de pouvoir édifier une tombe en l'honneur du mort. La tombe prouve l'honorabilité du mort; elle permet au

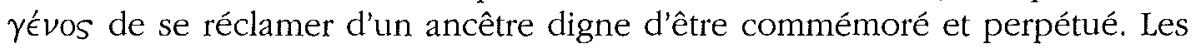
générations sont ici solidaires; la gloire des ancêtres est aussi et toujours celle des fils. En consacrant la dimension mémorable du mort, le tombeau permet à toute sa descendance de se prévaloir d'une origine exemplaire; il légitime la valeur des descendants. À la fin de l'rliade, si les Troyens tiennent absolument à célébrer les funérailles d'Hector, c'est tout à la fois pour honorer Hector et pour se réclamer de lui. Une terre où les morts resteraient sans sépulture serait une terre sans valeurs à défendre. Remarquons bien que s'il y a, dans la poésie homérique, une solution métaphysique au problème de la mort, un dépassement de la mort par la mémoire, cette solution est indissociable d'un système éthique ${ }^{28}$. Il convient ici de souligner une nuance qui n'a pas toujours été bien faite entre "mémoire » et «commémoration ». Les héros veulent survivre dans la mémoire des vivants. Mais la gloire à laquelle ils prétendent est loin de n'être qu'une pure célébrité : en aspirant à la gloire, le héros vise à accomplir un acte éthiquement exemplaire. Les noms de Thersite et de Clytemnestre sont bien passés à la postérité mais aucun héros ne souhaite devenir un Thersite ou une Clytemnestre. Le héros veut être commé-

27 Od., I, 214-220.

28 Cf. Vernant, op. cit. (n. 2), p. 52 et 94. 
moré comme une figure reconnue et acceptée par le groupe des vivants et non pas devenir un contre-modèle.

\section{La balance de mémoire et d'oubli ou la frontière de l'Hadès}

Faisons le point : dans la poésie homérique, la mémoire permet d'apporter une réponse au problème de la mort. En recevant une tombe, le mort ne bascule pas dans le vide du non-être mais devient sujet de commémoration, il existe dans et par la mémoire qui, en le célébrant, l'immortalise. Cependant, cette régénération du mort en figure mémorable n'est pas sans contrepartie. Il y a, dans la poésie homérique, une équation bien claire : si les mortels doivent se souvenir des défunts, les morts, eux, doivent être sans mémoire. Pourquoi ?

Il n'est pas aisé de répondre à une telle question. Mais on peut considérer qu'il s'agit d'un processus de compensation. Pour que la mémoire puisse agir pleinement comme un principe immortalisateur, il faut qu'elle soit la propriété exclusive des vivants. Si la commémoration n'était pas le seul moyen de dépasser la mort, le héros n'aspirerait pas tant à devenir figure de mémoire. Ceci implique une relation indissociable entre la vie et la mémoire.

Mais on peut souligner un autre aspect de cette problématique. L'immortalisation des morts dans le monde des vivants, le k $\lambda \dot{\epsilon}$ os qui leur est conféré, ne correspond aucunement à un prolongement de leur vie humaine. En devenant figure de mémoire, le héros ne gagne pas une nouvelle vie individuelle, ni même un nouveau destin, mais il devient un modèle qui ne peut être tel que parce qu'il a fini de vivre. Tant qu'il n'est pas achevé, aucun destin ne saurait être significatif ${ }^{29}$. Le héros ne pourra donc devenir objet de commémoration qu'après sa mort et, devenu tel, il ne saurait jouir d'aucune autre vie parallèle. Le mort ne doit pas pouvoir devenir autre chose que cette figure modèle que les vivants ont choisi d'immortaliser. L'immortalisation est ici une consécration. Pour que le mort puisse être pleinement et entièrement immortalisé par la mémoire des vivants, il faut que son destin se soit achevé : dans l'Hadès, il sera juste une ombre sans sensation ni mémoire, incapable d'agir à l'encontre de ce qu'il fut jadis, incapable de commettre un acte qui pourrait contredire ou échapper au souvenir qu'on a gardé de lui. L'inconscience des morts a ainsi, dans la poésie homérique, valeur de garantie; l'existence fantomatique du mort n'est que le négatif, la nécessaire contrepartie de sa consécration et de son immortalisation dans le monde des vivants.

On touche ici à un problème très compliqué : celui du rapport que cette conception homérique de la mort peut entretenir avec certaines pratiques religieuses et cultuelles de la réalité. Le problème n'est pas nouveau. E. Rohde l'avait déjà relevé à la fin du $\mathrm{xIx}^{\mathrm{e}}$ siècle, alors que l'histoire des religions en était à ses tout débuts.

29 Cette conception est encore largement répandue dans la Grèce du début du ve siècle, $c f$. par ex. les propos de Solon dans HDt., I, 32, 5 sq. et Eur., And., 100-103. 
Les «peuples primitifs » attribuent généralement aux «âmes » séparées du corps une puissance immense, invisible sans doute, mais d'autant plus effrayante; [...] Homère, au contraire, ne connaît aucune action des psychés dans le domaine du visible, et guère par conséquent de culte rendu aux âmes [...] Si l'on voulait en croire le seul Homère, les innombrables cultes locaux de la Grèce, avec leurs divinités liées à une étroite résidence, n'auraient pour ainsi dire pas existé : Homère les ignore presque complètement ${ }^{30}$.

Pour rendre compte de l'écart constaté, Rohde recourt à la thèse alors incontestée du miracle grec : dans cet âge primitif qui était celui des mythes et de l'irrationnel, Homère serait le précurseur et le premier représentant d'un esprit panhellénique soucieux de s'affranchir des craintes superstitieuses, surtout présentes dans les traditions cultuelles locales; la visée panhellénique de la poésie homérique est ici indissociable d'un effort de rationalisation ${ }^{31}$. Dans sa représentation du monde, des dieux et de la mort, Homère aurait inventé, d'après Rohde, « la fonction, véritablement poétique, sur laquelle repose tout l'idéalisme de l'art grec, et qui consiste à simplifier et à coordonner ce qu'il y avait de confus et d'exubérant dans les éléments dont il disposait $»^{32}$.

Aujourd'hui, si l'archéologie et les études homériques ont, chacune de leur côté et parfois ensemble, considérablement progressé, le problème n'en reste pas moins fondamentalement compliqué. Du côté de l'archéologie, le culte des morts et des héros n'apparaît plus comme l'une des plus anciennes et constantes traditions de la religion grecque : si l'on prend l'exemple des sépultures mycéniennes, on relève que les traces d'offrandes et d'activité cultuelle ne sont attestées qu'à partir de la fin du Géométrique (750-700) ${ }^{33}$. Certains ont supposé que l'émergence du culte héroïque à cette époque pouvait être une conséquence de la diffusion de la poésie homérique ${ }^{34}$; d'autres qu'il fallait, en revanche, établir un parallélisme entre l'émergence du culte héroïque au niveau des traditions locales et l'« émergence de l'épopée homérique » dans le contexte des grandes manifestations culturelles panhelléniques $^{35}$. Quelle que soit l'hypothèse choisie, le problème reste de comprendre pourquoi la poésie homérique ne reflète pas mieux la transformation des sentiments religieux des Grecs à l'époque du Géométrique récent.

30 E. Rohde, Psyché. Le culte de l'âme cbez les Grecs et leur croyance à l'immortalité, Paris, 1928 [or. all. 1894], p. 7 et pour la deuxième partie de la citation, p. 32.

31 Pour un développement de ce point de vue, cf. G. NAGY, Le meilleur des Acbéens, Paris, 1979 [or. angl. 1979], p. 148-152.

32 RoHDE, op. cit. (n. 30), p. 32.

33 Sur le problème de l'existence des cultes héroïques à l'époque homérique, voir F. DE POLIGNac, La naissance de la cité grecque, Paris, $1995^{2}$, p. 151-176, avec des indications bibliographiques, p. 190-192.

34 Ainsi L.R. Farneld, Greek Hero Cults and Ideas of Immortality, Oxford, 1921, p. 340342, et plus récemment J.N. Coldstream, Hero-Cults in the Age of Homer, in JHS, 96 (1976), p. 8-17.

35 Voir surtout NAGY, op. cit. (n. 31), p. 150 sq. 
Quoi qu'il en soit cependant de ce rapport compliqué à la réalité, il me suffit ici de démontrer, au sein de la poésie homérique, la cohérence du jeu de complémentarité qui se trouve établi entre une mémoire des morts dans le monde des vivants et un oubli de la vie dans l'Hadès. Cette cohérence révèle que la poésie des aèdes (dont l'lliade et l'Odyssée sont issues) a évolué en fonction de facteurs et d'impératifs qui lui étaient propres. De génération en génération, l'épopée aurait fini par privilégier l'image de l'Hadès dont elle avait le plus besoin. C'est par rapport à cette exigence idéologique interne que l'on peut comprendre pourquoi le pays des Lotophages est sur le chemin du héros une étape interdite. S'il apporte aux vivants qui le goûtent une forme de bonheur, le lôtos demeure un fruit redoutable : il empêche tout souvenir des morts. Quel sens donne-t-on au problème de la mort dans un pays où les vivants vivent d'oubli ?

Pour confirmer ces remarques qui restent théoriques, on peut rappeler le cas exceptionnel d'Aithalidès, le héraut des Argonautes. Apollonios de Rhodes évoque son destin singulier dans le premier livre de ses Argonauti$q^{4} e^{36}$. Fils d'Hermès, Aithalidès reçut de son père l'extraordinaire privilège de pouvoir choisir, entre tous les dons, celui qu'il voudrait. Contrairement au

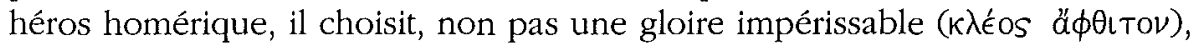

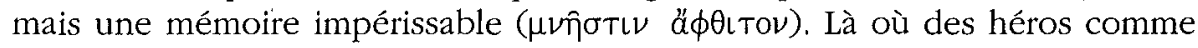
Achille et Hector aspirent à devenir inoubliables, Aithalidès préfère, lui, posséder une mémoire absolue, qui lui permettra de ne jamais rien oublier. Si, pour un héraut chargé de transmettre des messages, un tel choix est judicieux, il n'est pas sans conséquence: doté d'une mémoire éternelle qui gardera toujours le souvenir de la vie, Aithalidès ne saurait vraiment mourir. Son âme est destinée à revenir régulièrement dans le monde des vivants en parcourant un cycle perpétuel de naissances et de morts, « habitant tantôt sous la terre et tantôt parmi les vivants à la lumière du soleil $»^{37}$. La mémoire éternelle concédée à Aithalidès constitue une véritable transgression du code héroïque homérique : assuré de revenir dans le monde des vivants, Aithalidès n'a plus à accomplir l'exploit qui l'immortaliserait dans la mémoire des hommes à venir. Sa mémoire ne renvoie plus à des ancêtres exemplaires, elle ne vise plus à établir un lien entre les générations, mais elle enrichit Aithalidès d'un savoir lié à son propre passé et à ses propres expériences. Aithalidès échappe aux règles homériques de la mémoire et de l'oubli; c'est avec lui tout un système de valeurs qui est remis en cause; ce n'est pas un hasard si ce

36 A poll. Rh., Arg., I, $640 \mathrm{sq}$. Sur Aithalidès, cf. A. Ardizzoni, Echi pitagorici in Apollonio Rodio e Callimaco, in RFIC, 93 (1965), p. 257-267. Voir aussi Phéréc., 3 F 109 Jacoby ainsi que le commentaire de J.-P. Vernant, Mythe et pensée chez les Grecs, Paris, $1985^{10}$ [1965], p. 107-152.

37 Apoll. Rh., Arg., I, 646-648. 
héros a séduit les Pythagoriciens, adeptes d'une autre conception de l'audelà et prêts à croire que Pythagore était une réincarnation d'Aithalidès ${ }^{38}$.

\section{L'inutile mémoire des Sirènes}

L'épisode d'Elpénor et les inquiétudes de Télémaque permettent de mieux comprendre le piège que représentent le lôtos et le chant des Sirènes. La mémoire et l'oubli ont dans la pensée archaïque grecque un rôle bien précis et servent à articuler et à régler les relations entre vivants et morts. Le monde des Lotophages est inquiétant dans la mesure où l'oubli définit avant tout, dans la poésie homérique, la condition de morts devenus objets de mémoire. Dans le monde des Lotophages, la mort est niée. Par définition, les mangeurs d'oubli sont incapables d'honorer leurs morts et de leur édifier des tombes. Dans ce monde sans mémoire, tout se passe comme s'il n'y avait pas de distinction et de différence entre la vie et la mort.

Chez les Sirènes, la mémoire existe bien mais elle n'est qu'un, outil de séduction. Elle sert à charmer et relève uniquement de l'esthétique. D'un point de vue strictement héroïque ou humain, la mémoire des Sirènes est une contradiction. Dans le monde héroïque, la mémoire a une dimension religieuse et éthique : elle sert à célébrer et à perpétuer l'exemple de morts qui se sont rendus dignes d'être commémorés. Les tombes accordées aux morts sont là pour signifier ce pouvoir de la mémoire. Le charnier de cadavres sur le rivage de l'île des Sirènes révèle, en fait, un monde où les morts ne reçoivent aucune sépulture, où la mémoire, pour absolue qu'elle soit, n'implique cependant aucune reconnaissance des morts. Et c'est là pour le héros homérique quelque chose d'inacceptable. Dans le monde hérö̈que, une mémoire qui ne saurait pas se souvenir des morts perdrait toute dimension éthique. Se souvenir du passé, évoquer les guerres d'hier, c'est aussi pour les héros homériques rendre aux morts l'hommage qui leur est dû et tenter de donner, ainsi, un sens à la mort.

David Bouvier

Université de Lausanne

Section des Sciences de l'Antiquité

Faculté des Lettres

BFSH 2-4015

$\mathrm{CH}-1015$ DORIGNY

38 Selon un témoignage de Diog. LAËrce, VIII, 4-5, la légende circulait que Pythagore aurait été, dans une vie antérieure, Aithalidès lui-même. Empédocle aussi aurait prétendu à une mémoire post mortem, $c f$. EMP., $31 \mathrm{~B} 115,13-14 ; 117 \mathrm{D}-\mathrm{K}^{6}$. Voir aussi W. BurKERT, Les cultes à mystères dans l'Antiquité, Paris, 1992 [or. angl. 1987], et Lore and Science in Ancient Pythagoreanism, Cambridge, Mass., 1972. - Je tiens à remercier V. PirenneDelforge dont les suggestions m'ont été précieuses. 